\title{
Evaluación de alfacipermetrina para el control de ácaros en galli- nas ponedoras en condiciones de laboratorio
}

\author{
Evaluation of alpha-cypermethrin for the control of mites in laying hens under \\ laboratory conditions
}

\author{
Daisy Rodríguez García ${ }^{1,4}$, Yenisey García Ferrer ${ }^{1}$, Lissette Valdés Fraga ${ }^{1}$, Odalys \\ Correoso Mendoza ${ }^{1}$, Maylin González Navarrete ${ }^{2}$, Mario Valdés Rodríguez ${ }^{3}$
}

\section{Resumen}

\begin{abstract}
El objetivo del estudio fue evaluar dosis de alfacipermetrina para el control del ácaro Pterolichus obtusus en gallinas ponedoras en condiciones de laboratorio. El experimento se realizó en las naves experimentales del Instituto de Investigaciones Avícolas, Cuba, entre junio y julio de 2019. Se emplearon 115 gallinas ponedoras White Leghorn de 52 semanas de edad infestadas naturalmente con el ácaro. Se realizó un muestreo inicial a todas las aves para determinar los niveles de infestación y distribuirlas en grupos homogéneos. Se ensayaron cuatro dosis de alfacipermetrina $(1,2,5$ y $10 \mathrm{ml} / \mathrm{l})$ mediante baños de aspersión y se comparó con un grupo tratado con cipermetrina $(12 \mathrm{ml} / \mathrm{l})$ y un grupo control. Se efectuaron tres baños con frecuencia semanal. La efectividad de los tratamientos se evaluó entre 24 y 48 horas pos-tratamiento. La alfacipermetrina en dosis de 1 y $2 \mathrm{ml} / \mathrm{l}$ no resultó efectiva, mientras que en dosis de 5 y $10 \mathrm{ml} / \mathrm{l}$ fue altamente efectiva. La cipermetrina en dosis de $12 \mathrm{ml} / 1$ resultó altamente efectiva a partir del segundo baño.
\end{abstract}

Palabras clave: ponedoras, alfacipermetrina, ácaros plumícolas, Pterolichus obtusus

${ }^{1}$ Laboratorio de Investigación y Diagnóstico Aviar (LIDA) «Jesús Menéndez», Santiago de las Vegas, La Habana, Cuba

${ }^{2}$ Departamento de Prevención, Facultad de Medicina Veterinaria, Universidad Agraria de La Habana (UNAH), Mayabeque, Cuba

${ }^{3}$ Dirección de Investigación-Desarrollo, Empresa Química, Farmacéuticos y Plásticos, Grupo Empresarial Labiofam, La Habana, Cuba

${ }^{4}$ E-mail: daisyrg1978@gmail.com

Recibido: 13 de octubre de 2020

Aceptado para publicación: 13 de marzo de 2021

Publicado: 23 de junio de 2021

CLos autores. Este artículo es publicado por la Rev Inv Vet Perú de la Facultad de Medicina Veterinaria, Universidad Nacional Mayor de San Marcos. Este es un artículo de acceso abierto, distribuido bajo los términos de la licencia Creative Commons Atribución 4.0 Internacional (CC BY 4.0) [https:/ /creativecommons.org/licenses/by/4.0/deed.es] que permite el uso, distribución y reproducción en cualquier medio, siempre que la obra original sea debidamente citada de su fuente original 
The aim of the study was to evaluate doses of alphacypermethrin for the control of the mite Pterolichus obtusus in laying hens under laboratory conditions. The experiment was carried out in the experimental houses of the Poultry Research Institute, Cuba, between June and July 2019. In total, 115 White Leghorn laying hens, 52 weeks old, naturally infested with the mite, were used. An initial sampling was carried out on all the birds to determine the levels of infestation and distribute them in homogeneous groups. Four doses of alphacypermethrin $(1,2,5$ and $10 \mathrm{ml} / \mathrm{l})$ were tested by spray baths and compared with a group treated with cypermethrin $(12 \mathrm{ml} / \mathrm{l})$ and a control group. Three baths were carried out on a weekly basis. The effectiveness of the treatments was evaluated between 24- and 48-hour post-treatment. Alphacypermethrin in doses of 1 and $2 \mathrm{ml} / 1$ was ineffective, while in doses of 5 and $10 \mathrm{ml} / 1$ was highly effective. Cypermethrin at a dose of $12 \mathrm{ml} / \mathrm{l}$ was highly effective from the second bath.

Key words: layers, alphacypermethrin, feather mites, Pterolichus obtusus

\section{INTRODUCCIÓN}

Los ectoparásitos que afectan a las aves domésticas están representados por numerosas especies de ácaros y piojos (Roque, 2015). Las parasitosis en infestaciones intensas pueden ocasionar un cuadro debilitante (Torres-Cabra y Lagos-López, 2019), al interferir en la nutrición y descanso de las aves, con las consiguientes mermas en la producción (Quintero et al., 2010; Roque, 2015). Rodríguez et al. (2015) señalan que son frecuentes los casos en que las aves permanecen infestadas hasta el final de su ciclo productivo.

Tradicionalmente se emplean productos químicos para el control de estos artrópodos, especialmente los organofosforados y piretroides (Shane, 2010). Szczypel et al. (2003) indican que la cipermetrina es uno de los insecticidas más usados en Cuba y que con una correcta aplicación, garantiza hasta 98\% de efectividad. El empleo de plaguicidas químicos ha permitido una rápida y efectiva forma de solucionar estos problemas; sin embargo, la utilización inadecuada de estos productos conlleva a un desarrollo acelerado de resistencia de los parásitos externos a estos productos e incremento de los costos de producción (Hernández et al., 2007). Lister (2009) recomienda cambiar de principio activo cada cierto periodo de tiempo como medida para atenuar la aparición de resistencia. Shane (2010), de otra parte, refiere que existe un desarrollo de la resistencia a los piretroides y organofosforados sintéticos, en tanto que George et al. (2015) reportan resistencia de ácaros a plaguicidas como carbamatos y piretroides.

Estudios en Cuba se han llevado a cabo para determinar la eficacia de la alfacipermetrina en el control de piojos en gallinas ponedoras (Rodríguez et al., 2018); sin embargo, se desconoce su eficacia para el control de ácaros plumícolas en estas aves. Por todos es conocido que la aplicación del baño implica un estrés para las aves, sumado a que frecuentemente las infestaciones por ectoparásitos se presentan con la intervención de ácaros y piojos, por lo que se impone la necesidad de emplear productos que resulten eficaces para ambos grupos de ectoparásitos. Por tales razones, el objetivo 
del estudio fue evaluar dosis de alfacipermetrina para el control de ácaros plumícolas en gallinas ponedoras en condiciones de laboratorio.

\section{Materiales y Métodos}

El presente experimento se realizó en dos naves experimentales del Laboratorio de Investigación y Diagnóstico Aviar, perteneciente al Instituto de Investigaciones Avícolas, Cuba, durante los meses de junio y julio de 2019. Se emplearon dos naves (divididas en tres cuartones) con capacidad de 63 aves. Cada cuartón posee una fila o batería de siete jaulas, con mallas antipájaros para evitar la entrada de aves silvestres.

Se emplearon 115 gallinas ponedoras White Leghorn de 52 semanas de edad. Las aves fueron alojadas a razón de tres por jaula y la crianza se realizó según lo establecido por el Manual Tecnológico para Ponedoras y sus Reemplazos (Godínez et al., 2013). La alimentación fue a base de pienso ponedora fase II, a razón de $108 \mathrm{~g}$ por ave.

Las aves se identificaron con bandas en las alas. Se realizó un muestreo inicial a todas las gallinas conforme a lo referido por Rodríguez et al. (2018), extrayendo de 3 a 5 plumas de la cabeza, dorso, alas, zona de la cloaca, cara anterior del muslo y zona pectoral. Todas las aves se encontraban infestadas naturalmente por el ácaro Pterolichus obtusus. Para el diagnóstico aracnoentomológico y la clasificación del grado de infestación de los ácaros (Cuadro 1), se tuvo en cuenta los parámetros referidos por Rodríguez et al. (2015).

Las aves fueron distribuidas en grupos homogéneos de acuerdo con el grado de infestación inicial de $P$. obtusus. Cada grupo estuvo compuesto por 20 aves, con excepción del grupo 6 que fue de 15 gallinas (Cuadro 2). Se evaluó un concentrado emulsionable de alfacipermetrina al $10 \%$
Cuadro 1. Clasificación del grado de infestación por ácaros Pterolichus obtusus en gallinas según Rodríguez et al. (2015)

\begin{tabular}{ll}
\hline $\begin{array}{l}\text { Ácaros por } \\
\text { pluma }\end{array}$ & Diagnóstico \\
\hline 0 & Negativo \\
$1-5$ & Muy leve \\
$6-25$ & Leve \\
$26-50$ & Media \\
$51-100$ & Grave \\
$>100$ & Muy grave \\
\hline
\end{tabular}

(gabametrina), producida por Labiofam (Cuba). La dosis recomendada por el fabricante es de $1 \mathrm{ml} / 1$ de agua para el control de ácaros en mamíferos; pero se desconoce la dosis efectiva para el control de ácaros en aves.

Los tratamientos se aplicaron por medio de baños por aspersión empleando una mochila manual con capacidad para $16 \mathrm{~L}$. Se utilizó un volumen de solución de $100 \mathrm{ml}$ por ave. Se efectuaron tres baños con frecuencia semanal, realizándose una evaluación entre las 24 a 48 horas pos-tratamiento, contándose los ectoparásitos. El cálculo de la eficacia se realizó según la fórmula propuesta por la Resolución $\mathrm{N}^{\circ} 48$ del Mercosur (1997): \% $\mathrm{E}=((\mathrm{XT}-\mathrm{XD}) / \mathrm{XT}) * 100$, donde $\% \mathrm{E}$ es la eficacia en porcentaje, $\mathrm{XT}$ es la media de ectoparásitos del grupo testigo y $\mathrm{XD}$ es la media de ectoparásitos de cada grupo desparasitado. La interpretación de la eficacia considera que cuando se logra controlar más del $98 \%$ el tratamiento es altamente efectivo, entre $90-98 \%$ es efectivo, entre 80 $89 \%$ es moderadamente efectivo y menos del $80 \%$ es insuficientemente activo. 
Cuadro 2. Distribución de las gallinas por tratamiento según el nivel de infestación inicial por el ácaro Pterolichus obtusus

\begin{tabular}{ccc}
\hline Grupo & Tratamiento & $\begin{array}{c}\text { Grado de } \\
\text { infestación }\end{array}$ \\
\hline 1 & $\begin{array}{c}\text { Alfacipermetrina } \\
1 \mathrm{ml} / 1\end{array}$ & Grave \\
& Alfacipermetrina & Grave \\
2 & $2 \mathrm{ml} / 1$ & \\
& Alfacipermetrina & Grave \\
3 & $5 \mathrm{ml} / 1$ & \\
4 & Alfacipermetrina & Muy grave \\
& $10 \mathrm{ml} / 1$ & \\
5 & Cipermetrina & Muy grave \\
& $12 \mathrm{ml} / 1$ & \\
6 & Control & Muy grave \\
\hline
\end{tabular}

Los resultados fueron procesados mediante un análisis de varianza simple (ANOVA) para determinar si existen diferencias significativas entre los valores promedios de ácaros vivos con relación a los tratamientos aplicados y un test de rangos múltiples por Duncan para determinar diferencias entre tratamientos, con un nivel de confianza de 95\%. Para este análisis se utilizó el paquete estadístico Statgraphics Plus v. 5.1.

\section{Resultados y Discusión}

El ácaro presente en las gallinas del estudio era $P$. obtusus (Figura 1). Según Rodríguez (2017), P. obtusus y Megninia ginglymura son las especies de ácaros plumícolas más extendidas en Cuba. Las aves del estudio se encontraban parasitadas con niveles de infestación de hasta grave y muy grave. Estos valores coinciden con lo referi- do por Hernández et al. (2007) y Rodríguez et al. (2015) quienes explican que, en Cuba, las infestaciones por ácaros plumícolas en gallinas son muy abundantes con valores de positividad que oscilan entre 93.5 y $98.3 \%$; $y$ muchas veces se presentan en forma grave.

El confinamiento en jaulas limita las posibilidades de acicalamiento, favoreciendo de esta manera la multiplicación y dispersión de los parásitos externos en los galpones de producción y el aumento de los efectos negativos del parasitismo sobre las aves (Sánchez, 2004). Estos artrópodos, en infestaciones graves ocasionan intenso prurito, irritación, destrucción del plumaje y estados alérgicos generales, con una caída acentuada en la producción de huevos (Hernández et al., 2007; Rodríguez et al., 2018).

En el Cuadro 3 se presentan los resultados de los tratamientos antiparasitarios. Se evidenció que el número de ácaros vivos en tratamientos de los grupos 3, 4 y 5 fueron similares, pero significativamente menores que en los grupos 1 y 2 .

Teniendo en cuenta los resultados, se recomienda seleccionar el tratamiento donde se utilizó menos producto porque resulta más económico e implica menor contaminación

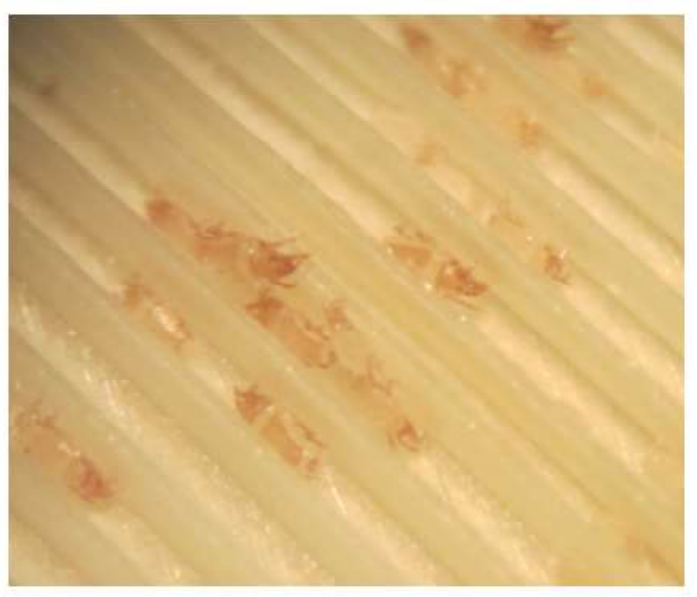

Figura 1. Ácaro Pterolichus obtusus parasitando plumas de gallinas ponedoras 
Cuadro 3. Valores promedios de ácaros Pterolichus obtusus vivos en los tratamientos con las soluciones de antiparasitarios en gallinas ponedoras

\begin{tabular}{|c|c|c|c|c|c|c|}
\hline \multirow[b]{2}{*}{ Baños } & \multicolumn{4}{|c|}{ Alfacipermetrina } & \multirow{2}{*}{$\begin{array}{c}\text { Grupo } 5 \\
\text { Cipermetrina } \\
(12 \mathrm{ml} / 1\end{array}$} & \multirow{2}{*}{$\begin{array}{c}\text { Grupo } \\
6 \\
\text { Contro }\end{array}$} \\
\hline & $\begin{array}{c}\text { Grupo } 1 \\
(1 \mathrm{ml} / \mathrm{l}) \\
\end{array}$ & $\begin{array}{c}\text { Grupo } 2 \\
(2 \mathrm{ml} / 1)\end{array}$ & $\begin{array}{c}\text { Grupo } 3 \\
(5 \mathrm{ml} / 1)\end{array}$ & $\begin{array}{c}\text { Grupo } 4 \\
(10 \mathrm{ml} / \mathrm{l})\end{array}$ & & \\
\hline 1 & 219.9 & 158.8 & 54.1 & 6.2 & 45 & 551.4 \\
\hline 2 & 171.3 & 169.9 & 7.9 & 2.5 & 9.8 & 760.7 \\
\hline 3 & 141.7 & 140.5 & 1.7 & 0 & 0 & 969.9 \\
\hline Media & $177.6^{\mathrm{a}}$ & $156.4^{\mathrm{a}}$ & $21.2^{\mathrm{b}}$ & $2.9^{\mathrm{b}}$ & $18.3^{\mathrm{b}}$ & 760.7 \\
\hline$\pm \mathrm{EE}$ & 22.8 & 8.6 & 16.5 & 1.8 & 13.6 & 120.8 \\
\hline
\end{tabular}

a,b Medias con letras desiguales en una misma fila difieren significativamente $(p<0.05)$

ambiental. Rodríguez (2019) explica que otro elemento a favor de la aplicación de tratamiento a partir del diagnóstico de infestaciones medias en adelante, es el factor económico. Sin embargo, estos resultados no son definitorios hasta tanto no se evalúe el producto en condiciones de producción, donde es muy posible, que la dosis realmente efectiva sea a razón de $10 \mathrm{ml} / 1$, o incluso superiores, si tenemos en cuenta, las experiencias obtenidas en el país con el empleo de los piretroides en la avicultura. En Cuba, la dosis efectiva de cipermetrina para el control de ectoparásitos en gallinas ponedoras es entre 10 y $15 \mathrm{ml} / 1$ (Szczypel et al., 2003; Hernández et al., 2007; Rodríguez et al., 2013).

Según Rodríguez (2019), la intención del tratamiento mediante aspersión no es erradicar los ectoparásitos, sino mantener bajas las poblaciones de los ácaros, a fin de que no se afecte su desempeño productivo. El uso de antiparasitarios debe quedar restringido a los casos necesarios con el fin de evitar el surgimiento de la resistencia de estos fármacos (Anziani et al., 2000). El antiparasitario es un recurso necesario, pero no renovable. La experiencia de más de cinco décadas ha de- mostrado que no existe antiparasitario «resistente» a la resistencia (Nari y Eddi 2002).

La alfacipermetrina en dosis de 1 y 2 $\mathrm{ml}$ resultó insuficientemente activa para el control del ácaro, pues la eficacia fue inferior al $80 \%$., mientras que la dosis de $5 \mathrm{ml}$ resultó altamente efectiva a partir del segundo baño y la dosis de $10 \mathrm{ml}$ resultó altamente efectiva desde el primer baño (Figura 2). Los resultados evidencian que el producto ensayado debe emplearse en dosis entre 5 y 10 ml. Por otro lado, la cipermetrina en dosis de $12 \mathrm{ml}$ resultó altamente efectiva a partir del segundo baño; resultado interesante pues demuestra que a pesar del prolongado tiempo que se ha utilizado en Cuba, aún existen poblaciones de ácaros sensibles a este producto.

\section{Conclusiones}

- La alfacipermetrina en dosis de 1 y 2 $\mathrm{ml} / 1$ no resultó efectiva para el control del ácaro Pterolichus obtusus en condiciones de laboratorio, pero fue altamente efectiva en dosis de 5 y $10 \mathrm{ml} / 1$. 


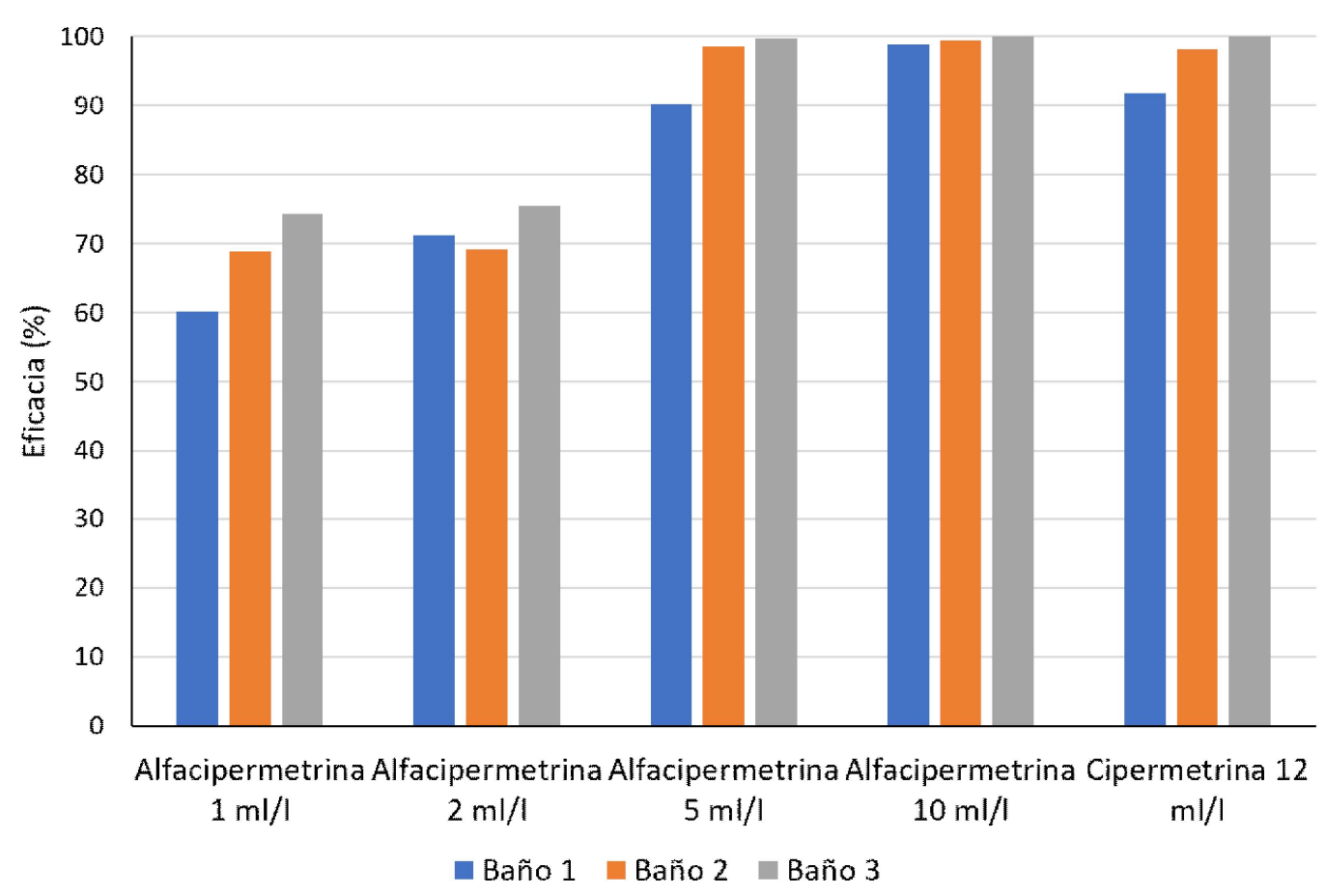

Figura 2. Eficacia (\%) de las dosis de alfacipermetrina y cipermetrina por baño realizado en gallinas ponedoras infestadas naturalmente con Pterolichus obtusus

- La cipermetrina en dosis de $12 \mathrm{ml} / \mathrm{L}$ resultó altamente efectiva a partir del segundo tratamiento.

\section{Literatura Citada}

1. Anziani O, Zimmermann G, Guglielmone A, Vasquez R, Suárez V. 2000. Resistencia a las ivermectinas de bovinos parasitados por Cooperia spp. Comunicación preliminar. Vet Arg 164: 280-281.

2. George DR, Finn RD, Graham KM, Mul MF, Maurer $V$, Moro CV, Sparagano $O A$. 2015. Should the poultry red mite Dermanyssus gallinae be of wider concern for veterinary and medical science? Parasite Vector 8: 178. doi:10.1186/s13071-015-0768-7
3. Godínez O, Pérez M, Colas M, Sardá R, Madrazo G. 2013. Manual tecnológico para la cría de aves. Reproductores ligeros y sus reemplazos. Ponedoras y sus reemplazos. La Habana, Cuba: Instituto de Investigaciones Avícolas. $110 \mathrm{p}$.

4. Hernández $M$, Lamarrendy $R$, Szczypel B, Morales Y, Temprana M. 2007. Evaluación de la efectividad de Bacillus thuringiensis cepa LBT-13 frente ectoparásitos de la gallina doméstica en una granja de ponedoras. Rev Cubana Cienc Avíc 31: 119-126.

5. Lister S. 2009. Desinfección y resistencia: irealmente la rotación es el camino a seguir? Industria Avícola 56: 18-20.

6. Mercosur. Resolución $N^{\circ} 48$ del 2 de mayo de 1997. Reglamentación técnica para licenciamiento y renovación de licencia de productos antiparasitarios de uso veterinario en Cuba. 
7. Nari A, Eddi CS. 2002. Control de la resistencia a los antiparasitarios a la luz de los conocimientos actuales. En: XI Congreso Mundial de Buiatría. Punta del Este, Uruguay.

8. Quintero MT, Itza M, Juárez G, Eleno A. 2010. Seasonality of Megninia ginglymura: a one-year study in a hen farm in Yucatan, Mexico. Trends in Acarology. p 537-538.

9. Rodríguez D. 2017. Ácaros de importancia veterinaria en la avicultura. En: IV Simposio de Biodiversidad Acarina. La Habana, Cuba.

10. Rodríguez D. 2019. Toma, envío de muestras e interpretación de los resultados parasitológicos en aves. En: VIII Jornada Científica Nacional de Avicultura, La Habana, Cuba.

11. Rodríguez D, Cruz C, Xuárez M, Correoso O, González, G, Pérez, R. 2018. Evaluación de la eficacia de diferentes dosis de alfacipermetrina y un concentrado emulsionable de Melaleuca en el control de piojos en gallinas ponedoras. Rev Cubana Cienc Avíc 42: 73-78.

12. Rodríguez D, Larramendy $R$, Varona E, Colás M, Reinaldo O, Villa JR, Morales Y, Cobas I, Rebollar R. 2015. Comportamiento de los parásitos externos en aves (Gallus gallus) de diferentes líneas puras. Rev Cubana Cienc Avíc 39: 5-12.

13. Rodríguez D, Xuárez M, Merino $A$, Larramendy $R$, Díaz $O$, Temprana $M$, Morales Y, Rebollar R. 2013. Evalua- ción de la eficacia de tres insecticidas de nuevo uso en la avicultura cubana para el control de ectoparásitos de las aves en condiciones de laboratorio. Rev Cubana Cienc Avíc 37: 25-32.

14. Roque E. 2015. Parasitología y enfermedades parasitarias en los animales domésticos. La Habana, Cuba: Félix Varela. 309 p.

15. Sánchez A. 2004. Principales enfermedades que afectan a las aves. En: Sánchez A, López A, Sardá R, Pérez M, et al. (eds). Salud y producción de las aves. La Habana: UNAH. p 185-465.

16. Shane SM. 2010. Manejo integrado de plagas: control eficaz de las moscas. Industria Avícola 57: 12-14.

17. Szczypel $B, \quad L a r r a m e n d y \quad R$, Hernández M. 2003. Evaluación de distintos insecticidas frente a ectoparásitos de la gallina doméstica. Rev Cubana Cienc Avíc 27: 121-124.

18. Torres-Cabra E, Lagos-López MI. 2019. Evaluación del aceite esencial de cilantro (Coriandrum sativum L.) sobre el ácaro rojo de aves Dermanyssus gallinae (De Geer, 1778) (Acari: Dermanyssidae) bajo condiciones de laboratorio. Cienc Tecnol Agropec Mosquera (Colombia) 20: 53-60.

19. Vial HJ, Traore M, Fairlamb AH, Ridley RG. 1999. Renewed strategies for drug development against parasitic diseases. Parasitol Today 15: 393-394. doi: 10.1016/s0169-4758(99)01524-0 